\title{
Hydroxychloroquine-induced Retinal Toxicity
}

KAYLENE L. CARTER 무, Medical student, MSEE; DIANA V. DO, MD, Professor, Byers Eye Institute, Department of Ophthalmology, Stanford University School of Medicine, Stanford, California, USA. Address correspondence to Dr. D.V. Do, Byers Eye Institute, Department of Ophthalmology, 2452 Watson Court, Palo Alto, California 94303, USA. E-mail: dianado@stanford.edu. Ethics board approval is not required because this is a single case report and no intervention had been made for research. The patient gave written informed consent to publish the material. J Rheumatol 2020;47:632; doi:10.3899/jrheum.190538

Hydroxychloroquine (HCQ) retinopathy is rare, with an estimated $0.33 \%$ incidence before 5-7 years of therapy, increasing to $2.1 \%$ by 15 years ${ }^{1}$.

A 62-year-old 59-kg woman with fibromyalgia and systemic lupus erythematosus presented to the ophthalmology clinic with blurry central vision in both eyes for several years. She had been taking $800 \mathrm{mg}$ of HCQ daily for the past 8 years. Optical coherence tomography imaging revealed bilateral disruption of the outer retinal structures and retinal pigment epithelium (RPE; Figure 1), and fundus autofluorescence imaging showed characteristic ring-shaped damage around the macula (Figure 2) known as bull's eye maculopathy, a late finding of HCQ-induced retinal toxicity. Additional manifestations may include perifoveal inner retinal thinning and displacement of inner retinal structures toward the $\mathrm{RPE}^{2,3}$. A maximum dose of $\leq 5 \mathrm{mg} / \mathrm{kg} /$ day is recommended by the American Academy of Ophthalmology, because greater doses can increase the risk of retinal toxicity, as in this case ${ }^{4}$. Other risk factors for retinal toxicity include advanced age, low body weight, tamoxifen use, and concomitant retinal, renal, or liver disease, which may necessitate more frequent screening ${ }^{4,5}$. In the early stages patients are typically asymptomatic, but as toxicity progresses patients can experience diminished visual acuity, peripheral vision, and night vision. Irreversible retinal changes may

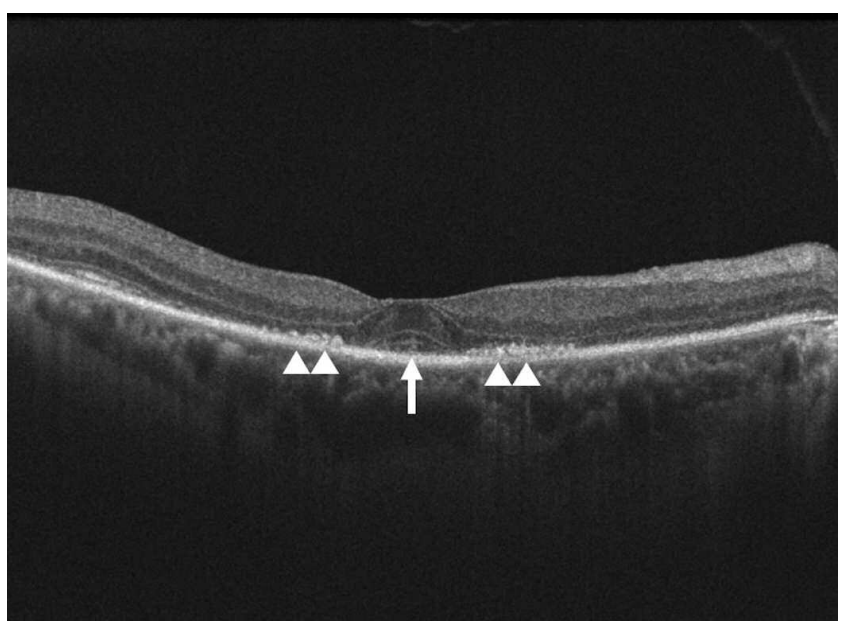

Figure 1. Optical coherence tomography of the patient's right eye. Bilateral disruption of the outer retinal structures and retinal pigment epithelium was detected in the parafoveal region (white arrowheads). Preservation of the central fovea outer retinal structures created an ovoid appearance of the central fovea (white arrow) known as the "flying saucer" sign of hydroxychloroquine retinopathy.

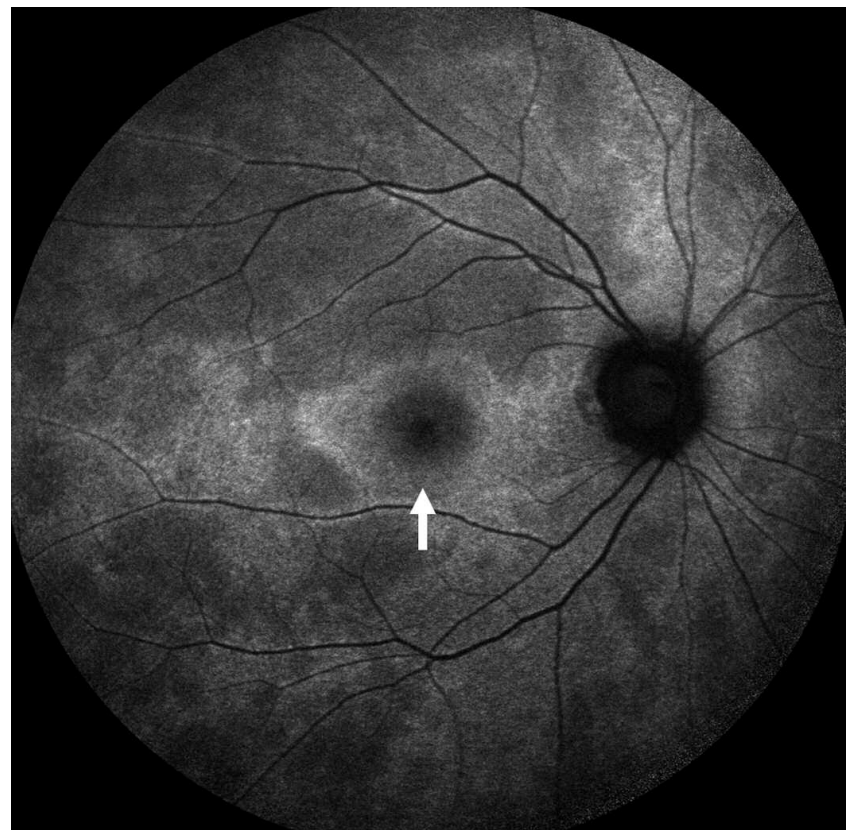

Figure 2. Fundus autofluorescence of the patient's right eye. An increased ring-shaped signal surrounding the macula (white arrow) displays retinal pigment epithelium degeneration and is a characteristic late finding in hydroxychloroquine retinopathy.

continue despite drug discontinuation, and therefore multimodal imaging measures are important to screen and identify signs of early toxicity. This patient was advised to immediately discontinue HCQ. Despite stopping the drug, progression of her central vision loss and the corresponding retinal and RPE abnormalities continued.

\section{REFERENCES}

1. Wolfe F, Marmor M. Rates and predictors of hydroxychloroquine retinal toxicity in patients with rheumatoid arthritis and systemic lupus erythematosus. Arthritis Care Res 2010;62:775-84.

2. Pasadhika S, Fishman G, Choi D, Shahidi M. Selective thinning of the perifoveal inner retina as an early sign of hydroxychloroquine retinal toxicity. Eye 2010;24:756-62.

3. Chen E, Brown D, Benz M, Fish R, Wong T, Kim R, et al. Spectral domain optical coherence tomography as an effective screening test for hydroxychloroquine retinopathy (the "flying saucer" sign). Clin Ophthalmol 2010;4:1151-8.

4. Marmor M, Kellner U, Lai Y, Melles R, Mieler W; American Academy of Ophthalmology. Recommendations on screening for chloroquine and hydroxychloroquine retinopathy (2016 Revision). Ophthalmology 2016;123:1386-94.

5. Peponis V, Kyttaris V, Chalkiadakis S, Bonovas S, Sitaras N. Ocular side effects of anti-rheumatic medications: what a rheumatologist should know. Lupus 2010;19:675-82. 